\section{Check for updates}

Cite this: Mater. Adv., 2022, 3,2026

Received 8th November 2021. Accepted 10th January 2022

DOI: 10.1039/d1ma01046h

rsc.li/materials-advances

\title{
Ultraflexible all-in-one supercapacitors with high capacitance and ultrastable cycle performance enabled by wood cellulose network $\dagger$
}

\author{
Shaoqiu Ke, $\ddagger^{\mathrm{ab}}$ Di Xie, $\neq^{\mathrm{b}}$ Kai Zhang, ${ }^{\mathrm{b}}$ Fangchao Cheng (D)*ab and Yiqiang Wu*a
}

\begin{abstract}
Herein, we demonstrate a structure-enabled strategy to construct an ultrastable, high-performance, ultraflexible all-in-one supercapacitor with a one-piece wood cellulose network/polyaniline/multiwalled carbon nanotube composite as both the electrodes and the separator. A cellulose network was used as the carrier and separator, and multiwalled carbon nanotube and polyaniline acted as conducting materials. Excellent flexibility was achieved by combining the cellulose network with a polyvinyl alcoholbased electrolyte. Natural directional channels in the cellulose network and the gradual distribution of the polyaniline and carbon nanotube within the structure enabled the construction of an integrated supercapacitor with reduced contact resistance between layers and improved electronic/ionic conductivity and stability. The supercapacitors resulted in a high specific areal capacitance of $0.71 \mathrm{~F} \mathrm{~cm}^{-2}$, as well as ultrastable cycle performance, which led to a $106.68 \%$ retention of the specific capacitance after 50000 loops at a current density of $3 \mathrm{~A} \mathrm{~g}^{-1}$. The current study provides a natureinspired strategy to rationally design and construct environmentally friendly high-performance and ultrastable energy storage devices.
\end{abstract}

\section{Introduction}

At present, excessive consumption of fossil fuels and consequential environmental pollution compel countries to search for the sustainable development of new energy and advanced technologies associated with energy storage. ${ }^{1}$ Supercapacitors, as one of the most important energy storage devices, can not only store energy like traditional batteries, but also have the advantages of ultra-high capacitance, high power density, rapid charging, long lifetimes, and excellent low-temperature performance and safety, and have been widely applied in electronics products, electric vehicles, military applications, and other industrial fields. ${ }^{2}$ Due to the popularity of electronic wearables, flexible supercapacitors (FSCs) are designed to operate without performance degradation under various strain conditions, such as bending, folding, and even twisting, which are particularly suitable for wearable electronics. ${ }^{3}$ In general, the performance of FSCs mainly depends on electrodes, and multifarious

\footnotetext{
${ }^{a}$ College of Material Science and Engineering, Central South University of Forestry and Technology, Changsha 410004, China. E-mail: fangchaocheng@gxu.edu.cn, wuyq0506@126.com

${ }^{b}$ Guangxi Key Laboratory of Processing for Nonferrous Metallic and Featured Materials, School of Resources, Environment and Materials, Guangxi University, Nanning 530004, China

$\dagger$ Electronic supplementary information (ESI) available. See DOI: 10.1039/d1ma01046h \$ These authors made equal contributions to this paper.
}

electrode materials have been used to construct FSCs, including graphene, ${ }^{4,5}$ carbon nanotube $(\mathrm{CNT}),{ }^{6}$ reduced graphene oxide, ${ }^{4,5}$ conductive polymers, ${ }^{7} \mathrm{MnO}_{2},{ }^{8}$ and other materials. ${ }^{9}$ Among them, natural renewable materials, such as cellulosic materials, have drawn much attention as flexible electrode matrixes due to their biodegradability and environment friendliness. Cellulosic materials ${ }^{10}$ (e.g., cellulose nanofiber, bacterial cellulose, cellulose nanocrystal, wood, and paper) have been combined with conducting polymers, ${ }^{5}$ carbon nanomaterials,${ }^{6}$ and metal oxides ${ }^{11}$ to form electrode materials for FSCs, resulting in high performance for energy storage and outstanding flexibility. However, FSCs prepared with cellulosic materials cannot take full advantage of the unique, naturally endowed structure of lignocellulosic biomass and cannot achieve high energy/power density, long cycle lives, and high mass loading simultaneously. ${ }^{12,13}$ Wood is a widely used material with unique porous hierarchical cellular structures originating from its natural growth. ${ }^{14}$ It is a promising concept to use wood as a template to produce electrode materials due to the naturally complex meshstructures of wood, which can provide a series of microreactors for other polymers or functional materials to form directional and functional composites. ${ }^{15,16}$ Recently, we prepared a highly conductive cellulose network/polyaniline composite as a promising electrode material by removing hemicellulose and lignin from wood while retaining the cellulosic skeleton structure of the wood. ${ }^{17}$ However, cellulose 
network-based conductive composites have poor flexibility due to the stiffness of the original wood. Therefore, further investigations are needed to realize the high energy/power density, long life cycles, and flexibility of naturally sourced, environmentally friendly cellulose network-based FSCs.

In addition to electrode materials, the electrolyte is an essential component that plays a crucial role in the performance of FSCs; it usually involves the liquid, gel phase, or allsolid-state forms. ${ }^{18}$ Due to the advantages of low cost, high reliability, and high ionic conductivity (about $10^{-4}$ to $10^{-1} \mathrm{~S} \mathrm{~cm}^{-1}$ ), gel electrolytes are commonly used in FSCs. ${ }^{19}$ Unfortunately, most FSCs using gel electrolytes (e.g., the polyvinyl alcohol (PVA)-acid system) exhibit poor structural integrity and low mechanical strength due to the nature of liquid gels. ${ }^{20}$ However, PVA-based gel electrolytes can be converted into robust, self-standing hydrogels through freezing-thawing cycles. $^{21}$ This may allow us to enhance the flexibility and mechanical strength of FSCs with the introduction of PVAbased gel electrolytes.

The structure design of FSCs is also of great importance in making full use of active electrode materials and facilitating ion and electron transport, which is particularly true when a thick electrode is utilized. Traditional FSCs have a multilayered structure that consists of two solid electrodes, a separator in between, and the electrolytes. ${ }^{22}$ However, the laminated configuration results in a large interface contact resistance because of the multiple interfaces among the layers, which hinders the fast charge and ion transport from the cathode to the anode. ${ }^{23}$ Furthermore, during the continuous bending or stretching cycles, the relative displacement between the different layers may lead to the electrode delamination of FSCs, which will undoubtedly reduce the performance of the device. ${ }^{23}$ In this case, decreasing the layer component number of FSCs and introducing an all-in-one structure into the FSCs may be beneficial to the stability of the FSCs and reduce the interface contact resistance, thus resulting in high performance for energy storage. As discussed above, the freezing-thawing treatment of PVA-based gel electrolytes can not only enhance the flexibility and mechanical properties of FSCs, but also allow for the assembly of all the components (electrodes, separator, and collectors) of FSCs without adhesive. Additionally, if the separator is substituted with an intermediate structure between electrodes constructed with gel electrolytes and a cellulose network, a novel all-in-one configuration of FSCs can be realized by fine-tuning the preparation process of the electrode materials, and the purpose of improving the energy storage performance and stability of FSCs would be expected to achieve by introducing the unique configuration and structure.

To verify this hypothesis, a novel type of all-in-one FSCs was developed based on a single-piece cellulose network originating from solid wood. In the structure of the FSCs, a piece of cellulose network/polyaniline/multiwalled carbon nanotube (CPM) composite acts as both the electrodes and separator, where the two surface parts containing polyaniline (PANI) and multiwalled carbon nanotube (MWCNT) act as the two electrodes, and the intermediate part of the composites between the two electrodes can be directly used as the separator. The PVAbased gel electrolytes can fix all components of the FSCs as well as act as an adhesive to fix the carbon cloth collectors. The cellulose network/polyaniline/multiwalled carbon nanotube (CPM) composites were synthesized by combining the chemical fractionation of solid wood, the immersion of MWCNT, in situ partial growth of PANI, and the coating of MWCNT onto the surface of the composites. The FSCs were assembled by the impregnation of the PVA-based gel electrolytes followed by freezing-thawing cycles. The FSCs exhibited high specific areal capacitance (up to $0.71 \mathrm{~F} \mathrm{~cm}^{-2}$ ), ultrahigh cyclic stability (about $106.68 \%$ retention after 50000 loops at $3 \mathrm{~A} \mathrm{~g}^{-1}$ ), promising ionic conductivity (up to $1.92 \times 10^{-2} \mathrm{~S} \mathrm{~cm}^{-1}$ ), and stable performance retention under various folding statuses. Moreover, the fabrication strategy of the all-in-one FSCs shows wide applicability, and different cellulosic materials, such as cotton cloth and cellulose paper, can be employed to construct FSCs with this method. The design strategy of all-in-one FSCs with a cellulose network provides a new direction for the preparation of integrated-structure, high-performance, safe, and reliable naturally sourced energy storage devices and may promote the sustainable and innovative development of wearable devices.

\section{Experimental}

\subsection{Materials}

Carboxylated multiwalled carbon nanotube with diameters of 10-20 nm was obtained from Jiangsu Xianfeng Nanomaterial Technology Co., Ltd (China). Phosphoric acid $\left(\mathrm{H}_{3} \mathrm{PO}_{4}, 98 \%\right)$ was purchased from Lianjiang Ailian Chemical Reagent Co., Ltd (China). Polyvinyl alcohol ( $\geq 97 \%$ ) was obtained from Tianjin Damao Chemical Reagent Factory (China). Potassium hydroxide $(\mathrm{KOH}, \geq 85 \%)$ was purchased from Chengdu Jinshan Chemical Reagent Co., Ltd (China). Acetic acid $\left(\mathrm{CH}_{3} \mathrm{COOH}\right.$, $\geq 99.5 \%$ ) was purchased from Chengdu Cologne Chemical Co., Ltd (China). Anhydrous ethanol ( $\geq 99.7 \%)$ was obtained from Tianjin Fuyu Fine Chemical Co., Ltd (China). Phytic acid (PA, $70 \%)$, sodium chlorite $\left(\mathrm{NaClO}_{2}, 98 \%\right)$, ammonium persulfate $(\geq 98 \%)$, and aniline $(\geq 99.5 \%)$ were provided by Shanghai Aladdin Bio-Chem Technology Co., Ltd (China). Balsa wood (Ochroma lagopus Swartz) was purchased from Zhuhai Dechi Technology Co., Ltd (China). All reagents were used as received.

\subsection{Fractionation, impregnation and polymerization processes}

Balsa Wood samples ( $2 \mathrm{~g}$ ) with dimensions of $15 \times 15 \times 1 \mathrm{~mm}$ were immersed in aqueous $\mathrm{KOH}$ solution $(2.5 \mathrm{M}, 50 \mathrm{~mL})$ at room temperature for $12 \mathrm{~h}$. Then, the mixture was refluxed for $3 \mathrm{~h}$ at $90{ }^{\circ} \mathrm{C}$ with magnetic stirring to remove most of the hemicellulose and part of lignin. Next, the sample was rinsed repeatedly with deionized water until the $\mathrm{pH}$ was equal to 7 . The treated samples were put into a $150 \mathrm{~mL}$ conical flask, and $65 \mathrm{~mL}$ deionized water, $0.5 \mathrm{~mL}$ glacial acetic acid, and sodium chlorite $(0.7 \mathrm{~g})$ were successively added to the flask. The 
mixture was heated at $75{ }^{\circ} \mathrm{C}$ for $1 \mathrm{~h}$, and more glacial acetic acid $(0.5 \mathrm{~mL})$ and sodium chlorite $(0.7 \mathrm{~g})$ were subsequently added. The above process was repeated three times to obtain white, purified wood samples. The as-prepared samples were rinsed repeatedly with deionized water until the $\mathrm{pH}$ equaled 7 and was stored in anhydrous ethanol to obtain a white cellulose network block.

The weight percent gain (WPG) of the cellulose network sample during the in situ polymerization process was calculated with the following eqn (1):

$$
\mathrm{WPG}=\left(\left(M_{1}-M\right) / M\right) \times 100 \%
$$

where $M$ is the weight of cellulose network, and $M_{1}$ is the weight of cellulose network/polyaniline/multiwalled carbon nanotube (CPM) composites.

Then, MWCNT solution $\left(0.15 \mathrm{mg} \mathrm{mL}^{-1}, 10 \mathrm{~mL}\right)$ was infiltrated into a cellulose network sample by vacuum filtration, and the sample was subsequently immersed in a solution containing aniline $(4.58 \mathrm{~mL})$, phytic acid $(6.59 \mathrm{~mL})$, and deionized water $(20 \mathrm{~mL})$ under vacuum for $0.3 \mathrm{~h}$. Subsequently, the ammonium persulfate solution, which was prepared by dissolving ammonium persulfate $(1.82 \mathrm{~g})$ in $10 \mathrm{~mL}$ deionized water, was slowly dropped into the above system containing the cellulose network sample at $5{ }^{\circ} \mathrm{C}$. The mixture was kept at $0{ }^{\circ} \mathrm{C}$ for $6 \mathrm{~h}$ to allow the in situ polymerization of the aniline monomer by diffusing APS into the cellulose network sample. After the in situ polymerization process, the sample was repeatedly rinsed with deionized water and anhydrous ethanol until the $\mathrm{pH}$ reached 7 , and then dried in a freeze-dryer for $24 \mathrm{~h}$. Narrow strips with widths of about $2.5 \mathrm{~mm}$ were cut off from four edges of the dried block to obtain the CPM composite sample with dimensions of $10 \times 10 \times 1 \mathrm{~mm}(L \times W \times H)$. To analyze the effect of MWCNT on the electrochemical performance of the composites, CP composites were also prepared using the same method without the infiltration of MWCNT.

\subsection{Fabrication of the supercapacitor prototype based on CPM block}

The PVA-based electrolyte was prepared by mixing $\mathrm{H}_{3} \mathrm{PO}_{4}(1 \mathrm{~g})$, deionized water $(10 \mathrm{~mL})$, and polyvinyl alcohol (PVA) powder $(1 \mathrm{~g})$ and then heating the mixture at $85{ }^{\circ} \mathrm{C}$ with magnetic stirring until it became clear; the solution was then kept at $85{ }^{\circ} \mathrm{C}$ for another $1 \mathrm{~h}$ without stirring. Then the electrolyte was fully immersed into the CPM blocks by vacuum impregnation. Subsequently, MWCNT was coated on the surface of the CPM blocks and adhered to the composites with the electrolyte. Different MWCNT loadings of $0.6,2.8,5$, and $7.2 \mathrm{mg} \mathrm{cm} \mathrm{cm}^{-2}$ resulted in corresponding electrode samples called $\mathrm{CPM}_{0}$, $\mathrm{CPM}_{1}, \mathrm{CPM}_{2}$, and $\mathrm{CPM}_{3}$, respectively. Two carbon cloth collectors were adhered to the surfaces of the $\mathrm{CPM}_{X}\left(\right.$ i.e. $\mathrm{CPM}_{0}, \mathrm{CPM}_{1}$, $\mathrm{CPM}_{2}$, and $\mathrm{CPM}_{3}$ ) samples with the electrolyte to assemble various $\mathrm{CPM}_{X}$ supercapacitors. The FSCs based on $\mathrm{CPM}_{X}$ composites were obtained after three cycles of freezing-thawing treatment with $12 \mathrm{~h}$ of freezing at $-20{ }^{\circ} \mathrm{C}$ and $3 \mathrm{~h}$ of thawing at room temperature in each cycle.

\subsection{Characterizations}

Scanning electron microscope (SEM). The wood, cellulose network, and CPM samples were characterized using SEM (Hitachi S-3400N, Tokyo, Japan), and the secondary electron images of the samples were collected with an acceleration voltage of $5 \mathrm{kV}$.

Field emission scanning electron microscope (FE-SEM). The wood, cellulose network, and CPM samples were also characterized using FESEM (Hitachi SU8220, Tokyo, Japan). And the secondary electron images of the samples were collected with an acceleration voltage ranging from $5 \mathrm{kV}$ to $15 \mathrm{kV}$.

Transmission electron microscopy (TEM). TEM images of MWCNT was collected using a FEI Tecnai G2 F30 TEM instrument (Thermo Fisher Scientific Corp., Hillsboro, OR, USA).

Brunauer-Emmett-Teller (BET) measurement. The porous structures of the wood, cellulose network and CPM samples were analyzed by a JW-BK300C three-station parallel automatic mesoporous microporous analyzer (Beijing Jingwei Gaobo Science and Technology CO., Ltd, Beijing, China).

Fourier transform infrared (FTIR) spectrum. FTIR spectra of the wood, cellulose network, PANI, and CPM samples were measured with a Nicolet iS 50 FT-IR instrument (Thermo Fisher Scientific Corp., Waltham, USA). All the samples were ground into 100 mesh, mixed with potassium bromide, and compressed into tablets. The spectra of each sample were recorded from 400 to $4000 \mathrm{~cm}^{-1}$ with 32 scans at a resolution of $4 \mathrm{~cm}^{-1}$.

Raman spectrum. Raman spectra of the cellulose network and CPM samples were measured with a inVia Reflex Reflex laser Raman spectrometer (Renishaw, Gloucestershire, UK). The laser wavelength was $633 \mathrm{~nm}$, and the test range was 200-3000 $\mathrm{cm}^{-1}$.

X-ray diffraction (XRD). The cellulose network, PANI, and CPM samples were ground into 100 mesh and characterized by a Smart Lab $3 \mathrm{~kW}$ X-ray diffractometer (Rigaku Corporation, Tokyo, Japan) equipped with Ni-filtered CuK radiation $(k=1.5406 \AA)$ at $40 \mathrm{kV}$ and $30 \mathrm{~mA}$. In this test, scattered radiation is recorded in the $2 \theta$ ranging from $5^{\circ}$ to $50^{\circ}$ at a scan rate of $5^{\circ} \mathrm{min}^{-1}$. The d-spacing of the lattice was calculated according to the Bragg equation (eqn (2)):

$$
d=n \lambda / 2 \sin \theta
$$

where $d$ is the lattice spacing of the sample, $\theta$ is the incident ray angle between the reflected line and the reflective crystal plane, and $\lambda$ is the wavelength. The relative crystallinity index $(\mathrm{Cr} I)$ of cellulose was calculated with the Segal method (eqn (3)):

$$
\mathrm{Cr} I=\left(\left(I_{002}-I_{\mathrm{am}}\right) / I_{002}\right) \times 100 \%
$$

where $I_{002}$ is the maximum intensity of the diffraction peak for the (002) crystal plane and $I_{\mathrm{am}}$ is the intensity of the amorphous background diffraction at $2 \theta=18^{\circ}$.

Electrochemical measurement. A three-electrode system at room temperature was employed to investigate the electrochemical properties of the samples electrodes in $1 \mathrm{M} \mathrm{H}_{2} \mathrm{SO}_{4}$ electrolyte. A silver-silver chloride $(\mathrm{Ag} / \mathrm{AgCl})$ electrode and $\mathrm{Pt}$ electrode were used as the reference and counter electrodes, 
respectively. The electrochemical properties of electrodes were analyzed with cyclic voltammetry (CV), galvanostatic chargedischarge (GCD) and electrochemical impedance spectroscopy (EIS) measurements using a CS $310 \mathrm{H}$ electrochemical workstation (Wuhan Corrtest Instruments Corp., Ltd, Wuhan, China). For three-electrode system, the voltage range for $\mathrm{CV}$ and GCD measurements were set to 0-0.8 V. CV measurement was conducted at scan rates of 2, 5, 10, 20, 50, and $100 \mathrm{mV} \mathrm{s}^{-1}$. GCD measurement was conducted at scan rates of $0.3,0.4,0.5$, 1 , and $2 \mathrm{~A} \mathrm{~g}^{-1}$. EIS analysis was performed with a perturbation of $10 \mathrm{mV}$ in the frequency range from $100 \mathrm{kHz}$ to $0.01 \mathrm{~Hz}$.

For the three-electrode tests, the area specific capacitance $C\left(\mathrm{~F} \mathrm{~cm}^{-2}\right)$ was calculated with eqn (4):

$$
C=\left(\int I \mathrm{~d} U\right) /(2 v S U)
$$

where $I(\mathrm{~A}), v\left(\mathrm{~V} \mathrm{~s}^{-1}\right), S\left(\mathrm{~cm}^{2}\right)$, and $U(\mathrm{~V})$ stand for the current, voltage sweep rate, working area of the electrode, and voltage window, respectively.

The electrochemical properties of the as-prepared supercapacitors were also characterized with CV and GCD methods. CV and GCD measurements were conducted with a potential window from $-0.6 \mathrm{~V}$ to $0.6 \mathrm{~V}$. The scan rates for $\mathrm{CV}$ and GCD measurement were same as those for the three-electrode tests. EIS analysis was also performed with the same settings as those for the three-electrode tests. The area specific capacitance $C_{\mathrm{s}}$ $\left(\mathrm{F} \mathrm{cm}^{-2}\right)$, the gravimetric specific capacitance $C_{\mathrm{g}}\left(\mathrm{F} \mathrm{g}^{-1}\right)$, and the bulk ionic conductivity $\sigma_{i}\left(\mathrm{~S} \mathrm{~cm}^{-1}\right)$ were calculated with eqn (5) and (6):

$$
\begin{aligned}
& C_{\mathrm{s}}=\left(\int I \mathrm{~d} U\right) /(v S U) \\
& C_{\mathrm{g}}=4(I \Delta t) /(m U)
\end{aligned}
$$

where $I(\mathrm{~A}), v\left(\mathrm{~V} \mathrm{~s}^{-1}\right), S\left(\mathrm{~cm}^{2}\right), U(\mathrm{~V}), \Delta t(\mathrm{~s})$, and $m(\mathrm{~g})$ stand for the current, voltage sweep rate, working area of the electrode, voltage window, discharge time, and total electrode sample weight, respectively. The ionic conductivity $\sigma_{i}\left(\mathrm{~S} \mathrm{~cm}^{-1}\right)$ was calculated with eqn (7):

$$
\sigma_{i}=d /(R S)
$$

where $d(\mathrm{~cm}), S\left(\mathrm{~cm}^{2}\right)$, and $R(\Omega)$ are the thickness, the area, and the bulk resistance, respectively. The energy density $E$ (W $\left.\mathrm{h} \mathrm{cm}^{-2}\right)$ and power density $P\left(\mathrm{~W} \mathrm{~cm}^{-2}\right)$ of the supercapacitors were calculated with eqn (8) and (9):

$$
\begin{gathered}
E=(1 / 2) \times C_{\mathrm{s}} \times U^{2} \\
P=E / t
\end{gathered}
$$

where $C_{\mathrm{s}}\left(\mathrm{F} \mathrm{cm} \mathrm{cm}^{-2}\right), U(\mathrm{~V})$, and $t(\mathrm{~h})$ are the areal specific capacitance, voltage window, and discharge time, respectively.

\section{Results and discussion}

The construction of all-in-one FSCs was realized in this study with the help of the straightforward cellular structure of the cellulose network, the controllable partial deposition of PANI, and the integrated assembly with PVA-based gel electrolytes. As graphically presented in Fig. 1a, a free-standing cellulose network retaining the structure and porosity of the original wood was produced by the chemical fractionation of solid wood. The three-layer structure of CPM composites was subsequently constructed through the immersion of MWCNT and in situ partial growth of PANI followed by cutting the four edges of the composites. The electrolyte was fully injected into the CPM composites by vacuum impregnation, and then MWCNT was coated on the surface to obtain $\mathrm{CPM}_{X}(X=0,1,2,3)$ composites. The $\mathrm{CPM}_{X}$-based FSCs were finally formed after introducing carbon cloth collectors and conducting three freezing-thawing cycles (Fig. 1a). The $\mathrm{CPM}_{X}$ composites endowed the FSCs with an integrated all-in-one structure containing both electrodes and a separator (Fig. 1b). The two surface parts contained active materials (i.e., MWCNT and PANI) that can act as the electrodes as well as an intermediate part of the composites between the two electrodes, where there is no distribution of active materials; thus, it can be directly used as the separator. The asprepared FSCs exhibited excellent flexibility, which allowed them to be wound around a finger and serve as a power source for wearable devices (Fig. 1c). Due to the extensive availability of wood, the $\mathrm{CPM}_{X}$-based FSCs possess scalable characteristics, and a scale-up sample of CPM composites with dimensions of $10 \times 12 \times 2 \mathrm{~cm}$ is presented in Fig. 1d. The CPM electrode materials that featured super flexibility can endure various forms of deformation such as bending, curling, twisting, and folding (Fig. 1e). Tensile tests were performed on the all-in-one FSCs to verify the adhesion strength between the current collector, MWCNT layer and CPM composites. The results showed that the all-in-one supercapacitor exhibited a promising tensile strength of $169.70 \mathrm{KPa}$ (Fig. S1, ESI $\dagger$ ). Moreover, the present preparation strategies of the $\mathrm{CPM}_{X}$ electrodes as well as the corresponding FSCs showed excellent applicability on other cellulose materials commonly used in daily life, such as cotton cloth and cellulose paper. The CPM composites based on cotton cloth and cellulose paper can also produce a threelayer structure by the in situ partial growth of PANI (Fig. 1f), which can be utilized as an electrode material for the all-inone FSCs.

Fig. 2 demonstrates the morphologies and characterizations of products in various stages of the preparation process of the CPM composites. A white cellulose network block with high porosity (Fig. 2a) was firstly produced by the chemical fractionation of the solid wood with alkali and sodium chlorite, which can dissolve hemicellulose and decompose lignin into watersoluble compositions through the reactions of phenolic and alcoholic hydroxyls. ${ }^{24}$ The fractionation process can enhance the nanostructure of the cellulose network by removing most of the hemicellulose and lignin distributed between the cellulose microfibrils, which was confirmed by the disappearance of the characteristic peaks of hemicellulose and lignin in the Fouriertransform infrared spectra (Fig. S2, ESI $\dagger$ ). Cellulose microfibril bundles were observed on the cross-section of the cellulose network (Fig. 2b). The process can also deconstruct the structures of pit pairs on the wood cell wall to produce micron-scale holes connecting the adjacent wood cells (Fig. S2, ESI $\dagger$ ). 
(a)

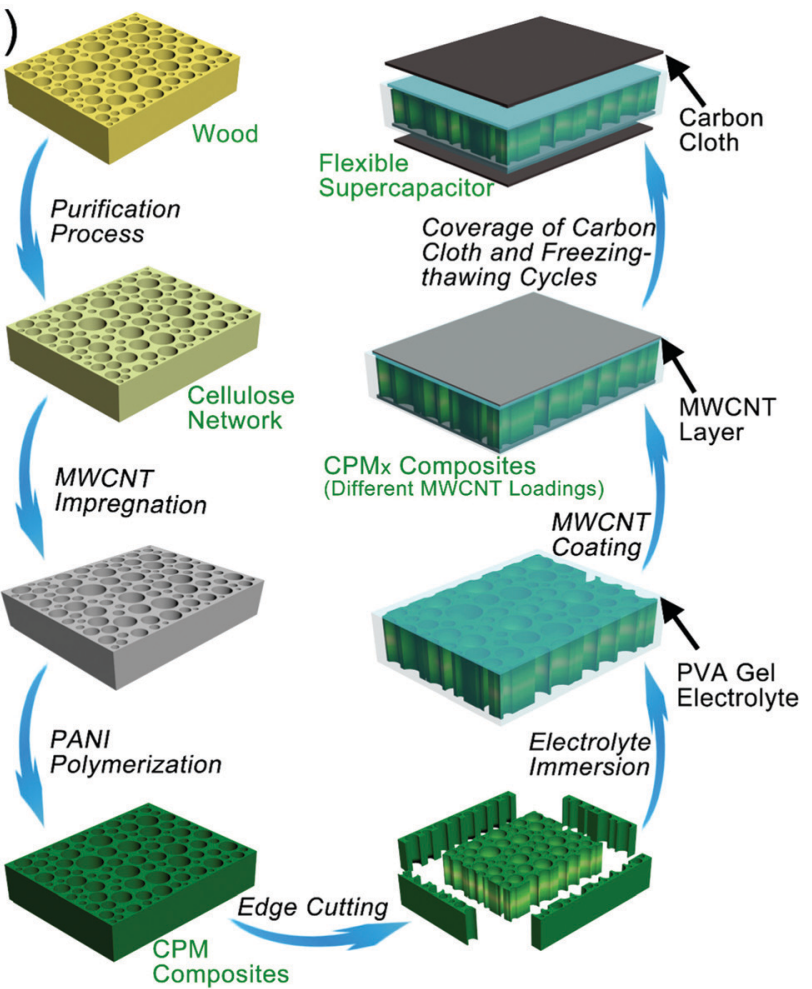

(b)

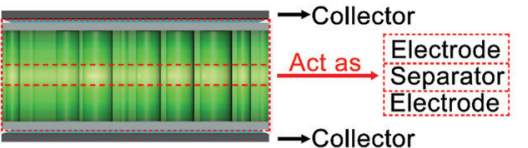

(c) All-in-one FSCs

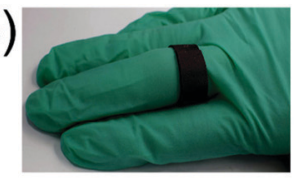

Flexible

(e)

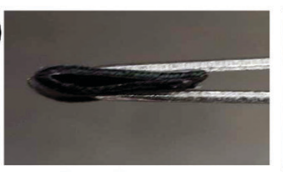

Bending

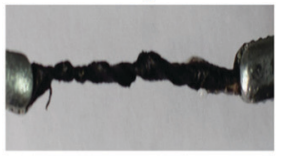

Twisting

(f)

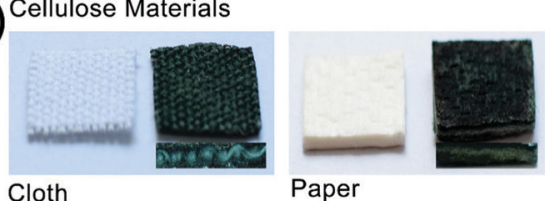

Fig. 1 Construction procedures and characteristics of the CPM ${ }_{X}$ composites and the corresponding FSCs: (a) schematic diagram of the preparation process of the all-in-one FSCs, (b) the structure of the all-in-one FSCs, (c) flexibility of the CPM -based FSC, (d) scalable CPM composites, (e) various deformation forms of $\mathrm{CPM}_{X}$ composites, and (f) CPM composites based on different cellulose materials.
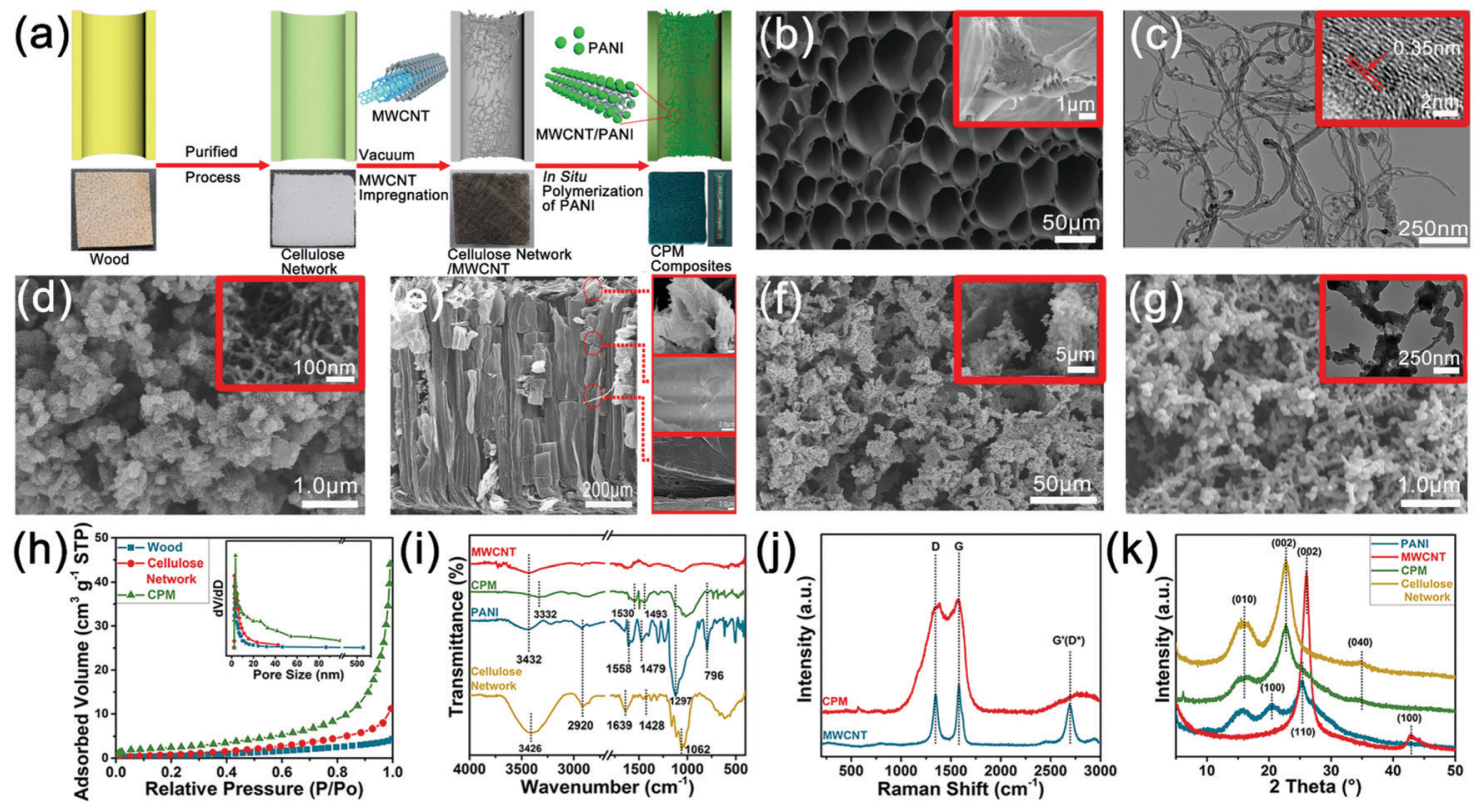

Fig. 2 The morphologies and characterizations of various products during the preparation process of the CPM composites: (a) schematic diagrams of the wood cell lumen during the preparation process of CPM composites and the photographs of the corresponding samples, (b) SEM images for the cellulose network, (c) TEM images for MWCNT, (d) FESEM images for PANI, (e) SEM images for the longitudinal section of the cellulose network/MWCNT sample, (f) SEM images for the cross section (surface) of CPM composites, (g) SEM and TEM images for the MWCNT/PANI complex in CPM composites, (h) nitrogen adsorption-desorption curve and the pore size distribution of wood, cellulose network, and CPM composites, (i) FTIR spectra of the MWCNT, cellulose network, PANI, and CPM composites, (j) Raman spectra of MWCNT, PANI, and CPM composites, and (k) X-ray diffraction patterns of the MWCNT, cellulose network, PANI, and CPM samples. 
The enhanced hierarchical porous structure makes the cellulose network an excellent matrix for further compounding and modification, in order to produce electrode materials and energy storage devices. ${ }^{17}$ It is reported that the introduction of MWCNT can reduce the ion diffusion impedance of PANI by forming a continuous phase for charge transfer, thereby improving the capacity, stability, and functional characteristics of the PANI-based electrode. ${ }^{25}$ In this study, MWCNT was firstly infiltrated into the cellulose network before the in situ polymerization of PANI to construct a cross network with PANI in the next step. SEM results showed a gradient distribution of MWCNT on the inner wall of the wood cell lumen, and the MWCNT distribution gradually decreased from the surface to the center (Fig. 2e and Fig. S3a-c, ESI $\dagger$ ). TEM images illustrate the multiple-wall structure of MWCNT (Fig. 2c). It should be noted that a low mass loading of MWCNT $\left(0.6 \mathrm{mg} \mathrm{cm}^{-2}\right)$ was employed to avoid a possible short circuit when the composites were used as the integrated electrodes for the FSCs (Fig. S2, ESI $\dagger)$. The as-prepared cellulose network/MWCNT complex was then impregnated with aniline monomers, and the in situ deposition of PANI was realized through the chemical oxidative polymerization of aniline. ${ }^{26}$ Extremely dense distribution of PANI was observed on the cross-section of the CPM composites (Fig. 2f). Additionally, inside the cell lumen, PANI was found to be intensively deposited on the MWCNT surface to form a cross-network and anchor the MWCNT to the inner cell wall (Fig. 2g). Interestingly, the PANI deposition on the inner cell wall presented a gradual change from the surface to the interior that can be seen from the longitudinal section of the sample (Fig. 2a), and the mass loading and distribution of PANI in the CPM composites can be tuned by varying the reaction time and PA concentration (Fig. S2, ESI $\dagger$ ). Thus, a three-layer structure of the CPM composites can be formed through regulating the mass loading of PANI (Fig. S2, ESI $\dagger$ ); the corresponding weight percent gain for PANI ranged from $41.0 \%$ to $80.4 \%$. The layered structure enabled the subsequent preparation of integrated electrode materials and the construction of all-in-one FSCs.

The influence of the fractionation and in situ polymerization process on the porosity of the wood matrix was investigated by comparing the BET surface area and pore volume of the wood, cellulose network, and CPM samples (Fig. 2h). The BET surface area of the cellulose network was $3.382 \mathrm{~m}^{2} \mathrm{~g}^{-1}$, which was slightly higher than $1.817 \mathrm{~m}^{2} \mathrm{~g}^{-1}$ of wood, indicating that the fractionation process can remove most of the hemicelluloses and lignin fractions of wood and enhance its porosity and microstructure. ${ }^{27}$ By comparison, the CPM composite exhibited a much higher BET surface area $\left(8.773 \mathrm{~m}^{2} \mathrm{~g}^{-1}\right)$ than that of the cellulose network and wood samples. Moreover, the comparison of pore size distribution curves (Fig. 2h) illustrated that the CPM composite possessed a much higher pore volume $\left(0.065 \mathrm{~cm}^{3} \mathrm{~g}^{-1}\right)$ relative to that of the cellulose network $\left(0.017 \mathrm{~cm}^{3} \mathrm{~g}^{-1}\right)$ and wood $\left(0.006 \mathrm{~cm}^{3} \mathrm{~g}^{-1}\right)$. Compared with cellulose network and wood, the pore size in the CPM composite showed a wider distribution within the range of micropore and mesoporous structures (Fig. 2h). These results suggest that the cellulose network had abundant nanoscale pore distribution despite its specific surface area being small. The introduction of PANI and MWCNT enriched the pore structure of the CPM composites, especially the micropores and mesopores.

The interactions of cellulose, PANI, and MWCNT in the CPM composites were analyzed by FTIR, Raman spectra, and XRD analyses. Most of the characteristic peaks for cellulose, PANI, and MWCNT were present in the FTIR spectra of the CPM composites (Fig. 2i). Compared with three respective spectra, red shift and a decreased intensity of the $\mathrm{O}-\mathrm{H}$ stretching absorption peak can be observed in the spectrum of the CPM (Fig. 2i), which indicated that the incorporation of PANI and MWCNT partly weakened the native hydrogen bonding of the cellulose, and that these three components formed a new hydrogen bonding network in the CPM composites. ${ }^{28}$ Consistent with the above deduction, XRD patterns confirmed the decrease of cellulose crystallinity with the introduction of PANI and MWCNT; the relative crystallinity of the cellulose decreased from $60.23 \%$ for the cellulose network to $54.97 \%$ for the CPM composites (Fig. 2k). The decrease in crystallinity may be mainly attributed to the infiltration of aniline monomers into the cellulose microstructure and the subsequent in situ formation of PANI. ${ }^{28}$ The Raman spectra of the CPM composites revealed high similarity with those of PANI and MWCNT, indicating the successful in situ polymerization of PANI around MWCNT (Fig. 2j). The Raman spectra also confirmed that the incorporation of PANI had no obvious influence on the multiwall structure of MWCNT.

During the preparation process of the CPM composites, MWCNT was infiltrated into the cellulose network to form an ionic conductive complex with PANI, and an improvement on the electrochemical performance was expected to be achieved. To test this hypothesis, two kinds of composites were prepared based on the cellulose network and PANI for comparison: CPM composites with MWCNT and cellulose network/PANI (CP) composites without MWCNT. The effects of the incorporation of MWCNT on the electrochemical performance of the electrodes were evaluated by three-electrode tests with CV, GCD, and electrochemical impedance spectroscopy methods (Fig. 3). The cyclic voltammetry curves (Fig. 3a-c) at different scan rates confirmed the evident capacitive nature of the CP and CPM composite electrodes. Two pairs of redox peaks for PANI can be observed in the CV curves at a scanning rate of $2 \mathrm{mV} \mathrm{s}^{-1}$ (Fig. 3c), which should be attributed to the embedding and stripping of $\mathrm{H}^{+}$from $\mathrm{H}_{2} \mathrm{SO}_{4}$ electrolytes in PANI, which was also the main source of capacitance. ${ }^{29,30}$ The cellular structures in the cellulose network can act as microreactors for the in situ preparation of PANI along the inner wall of the wood cell lumen. Considering the fact that the $\mathrm{H}^{+}$doping degree of PANI mainly determined its conductivity, ${ }^{31}$ the ion may primarily transmit along the cell lumen in this case. Thus, the microporous structure formed by the cellulose network and PANI can provide ion channels for electrolyte penetration and ion transmission when the composites are used as electrode materials. Compared to the CP electrode, a more visible curvature of the CV curve for the CMP electrode implied its stronger 

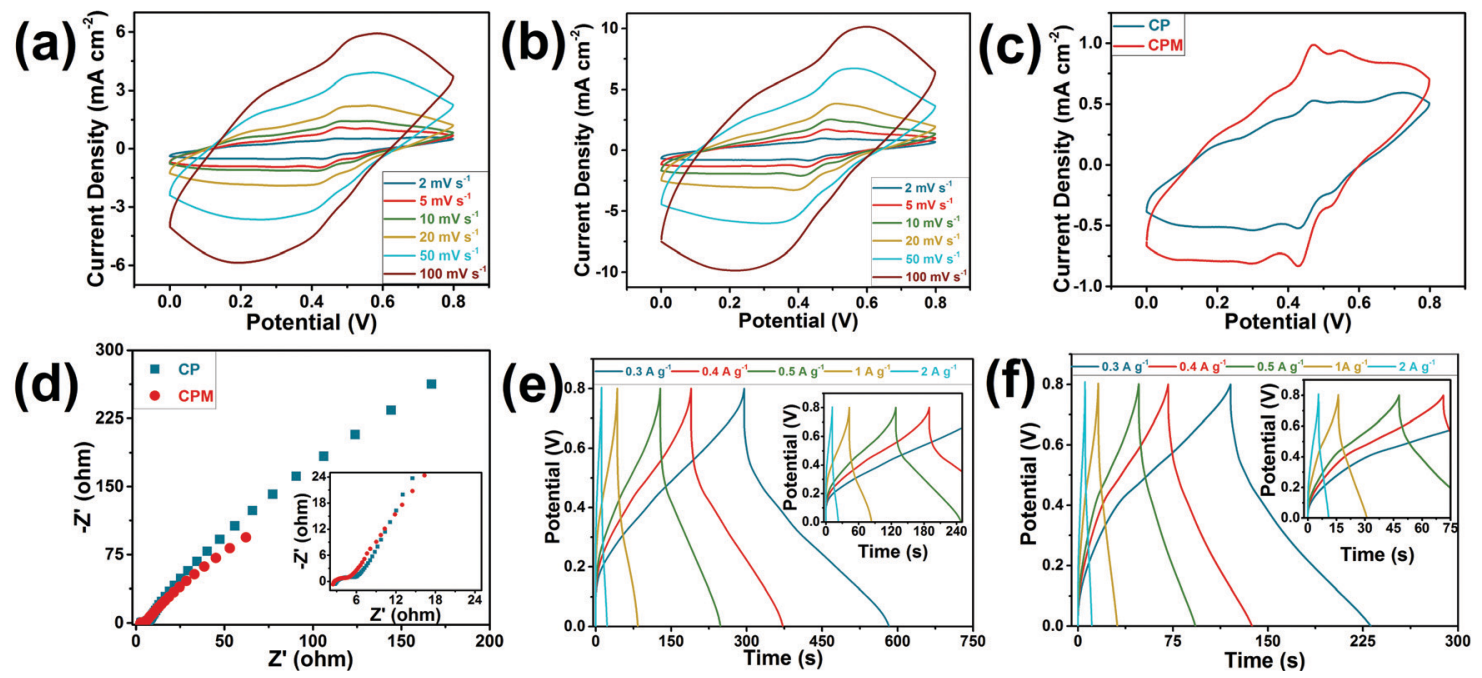

Fig. 3 Electrochemical performance of the wood-derived electrode materials in a three-electrode test. CV curves for (a) CP and (b) CPM electrodes under various sweep rates. (c) CV curves of the CP and CPM electrodes at a sweep rate of $2 \mathrm{mV} \mathrm{s}^{-1}$. (d) Nyquist diagrams for CP and CPM. GCD curves for (e) CP and (f) CPM electrodes under various current densities.

pseudocapacitive feature, and the comparison of the integral areas demonstrated the much higher capacitance of the CPM electrode. The CPM electrode provided a promising areal specific capacitance of $0.27 \mathrm{~F} \mathrm{~cm}^{-2}$ at a scan rate of $2 \mathrm{mV} \mathrm{s}^{-1}$ in the three-electrode test, while the $\mathrm{CP}$ electrode resulted in an areal specific capacitance of $0.14 \mathrm{~F} \mathrm{~cm}^{-2}$ at the same scan rate; this was mainly attributed to the incorporation of MWCNT in the CPM electrode, which increases the specific surface area of the sample and causes the chain of PANI molecules linked together to have no contact with each other as well as the corresponding enhanced electron transfer and electrochemical reactivity. ${ }^{29}$ The Nyquist diagram (Fig. 3d) of the CPM electrode showed a lower interface resistance than that of the CP electrode $(2.66 \mathrm{Ohm} v s .3 .00 \mathrm{Ohm})$, indicating the higher electronic/ion transmission rate for the CPM electrode. ${ }^{30}$ The bulk ionic conductivities of the $\mathrm{CP}$ and CPM electrodes were also determined with the EIS method (Fig. 3d). The CPM electrode showed a slightly higher ionic conductivity of $3.76 \times$ $10^{-2} \mathrm{~S} \mathrm{~cm}^{-1}$ than that of the $\mathrm{CP}$ electrode $(3.33 \times$ $10^{-2} \mathrm{~S} \mathrm{~cm}^{-1}$ ), which was consistent with the above interfacial resistance results. The galvanostatic charge-discharge curves for the CP and CPM electrodes at different current densities (Fig. 3e and f) exhibited approximately symmetrical triangular shapes, which reflected their promising conductivity properties. The CPM electrode exhibited much better gravimetric specific capacitances $\left(186.58 \mathrm{~F} \mathrm{~g}^{-1}\right.$ vs. $\left.110.52 \mathrm{~F} \mathrm{~g}^{-1}\right)$ than that of the CP electrode. In addition, the Coulombic efficiency for the CPM electrode reached $93.9 \%$, which confirmed its high electronic/ion transmission rate. ${ }^{30}$ The promising conductivity and specific capacitance of the as-prepared CPM composite makes it an important candidate for electrode materials. However, it is also suitable to act as the integrated electrode material for all-in-one supercapacitors when considering its outstanding advantage of possessing an integrated structure.
Given the integrated structure of the CPM composites, we proposed that an integrated all-in-one FSC device can be obtained based on a single CPM block. Corresponding to the electrode-separator-electrode structure of the traditional supercapacitor, two surface parts of the CPM block acted as the electrodes in the integrated all-in-one FSC, and the middle part acted as the separator (Fig. 1b). Additionally, the CPM composites showed uneven and porous surfaces on the cross sections (Fig. 2f). In order to obtain flat surfaces of the CPM composite electrodes and improve their electrochemical energy storage performance, different loadings of MWCNT were coated on the surfaces of the CPM composites and adhered to the surface with electrolytes to form the $\mathrm{CPM}_{X}(X=0,1,2$, and 3) integrated electrode materials. The integrated FSC was then assembled by the coverage of carbon clothes on a single $\mathrm{CPM}_{X}$ block as the collectors and the subsequent freezingthawing cycles of PVA-based gel electrolytes. The freezingthawing treatment improved the physical cross-linking degree of the PVA-based gel electrolytes, ${ }^{32}$ and the gel electrolytes were thus converted into ultraflexible solid materials, resulting in an excellent flexibility of the as-prepared FSCs (Fig. 1b).

Fig. 4 presents the electrochemical properties of the integrated all-in-one FSCs based on the $\mathrm{CPM}_{X}$ composites. The CV curves of all the FSCs exhibited a rectangular shape (Fig. 4a), indicating their promising capacitive behavior. The characteristic redox peaks of PANI can also be observed on the CV diagrams of all the FSCs at a scan rate $2 \mathrm{mV} \mathrm{s}^{-1} \cdot{ }^{37}$ The specific areal capacitance of the FSC based on $\mathrm{CPM}_{0}$ (i.e. without the coating of MWCNT) was $0.12 \mathrm{~F} \mathrm{~cm}^{-2}$ at a sweep rate of $2 \mathrm{mV} \mathrm{s}^{-1}$. The FSCs based on the $\mathrm{CPM}_{X}$ composites with the coating of MWCNT $\left(\mathrm{CPM}_{1}, \mathrm{CPM}_{2}\right.$, and $\left.\mathrm{CPM}_{3}\right)$ showed much higher specific areal capacitance of up to $0.71 \mathrm{~F} \mathrm{~cm}^{-2}\left(\mathrm{CPM}_{2}\right)$; the capacitance increased first and then decreased as the mass loading of MWCNT increased from $0 \mathrm{mg} \mathrm{cm}^{-2}$ to $7.2 \mathrm{mg} \mathrm{cm}^{-2}$ 

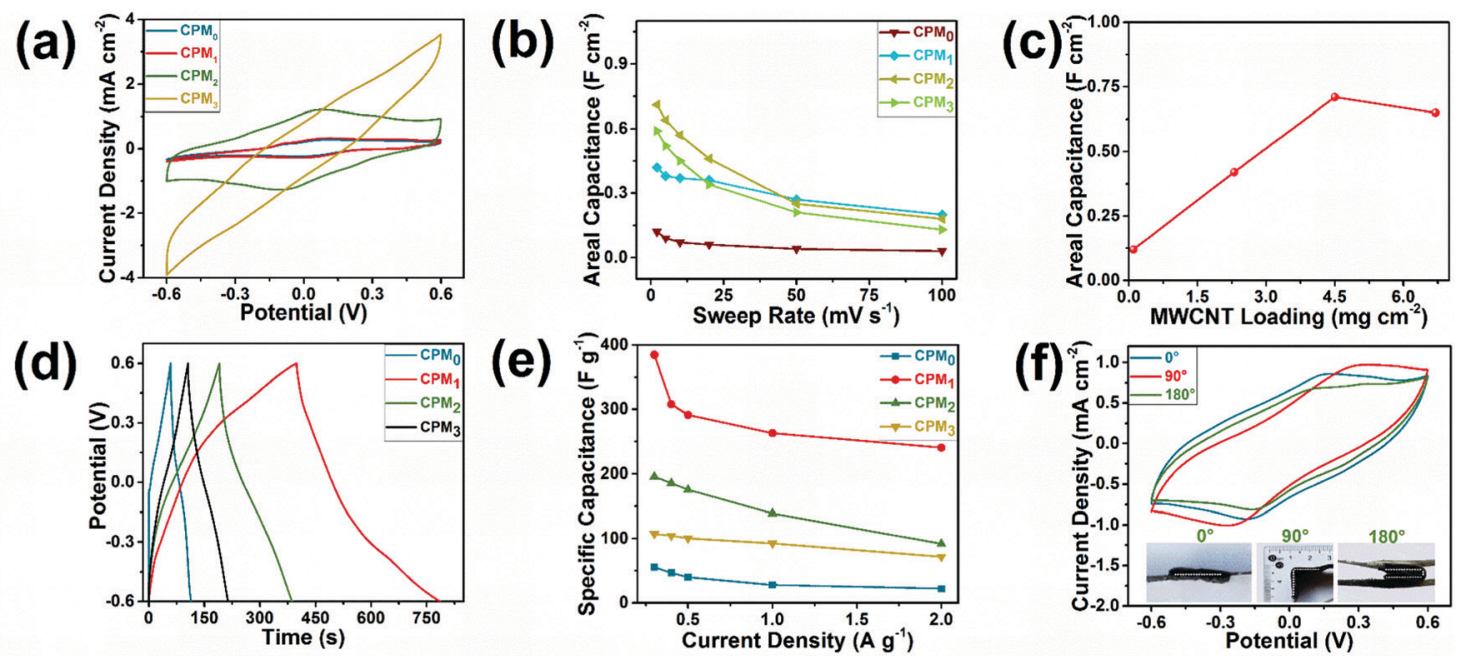

(e)
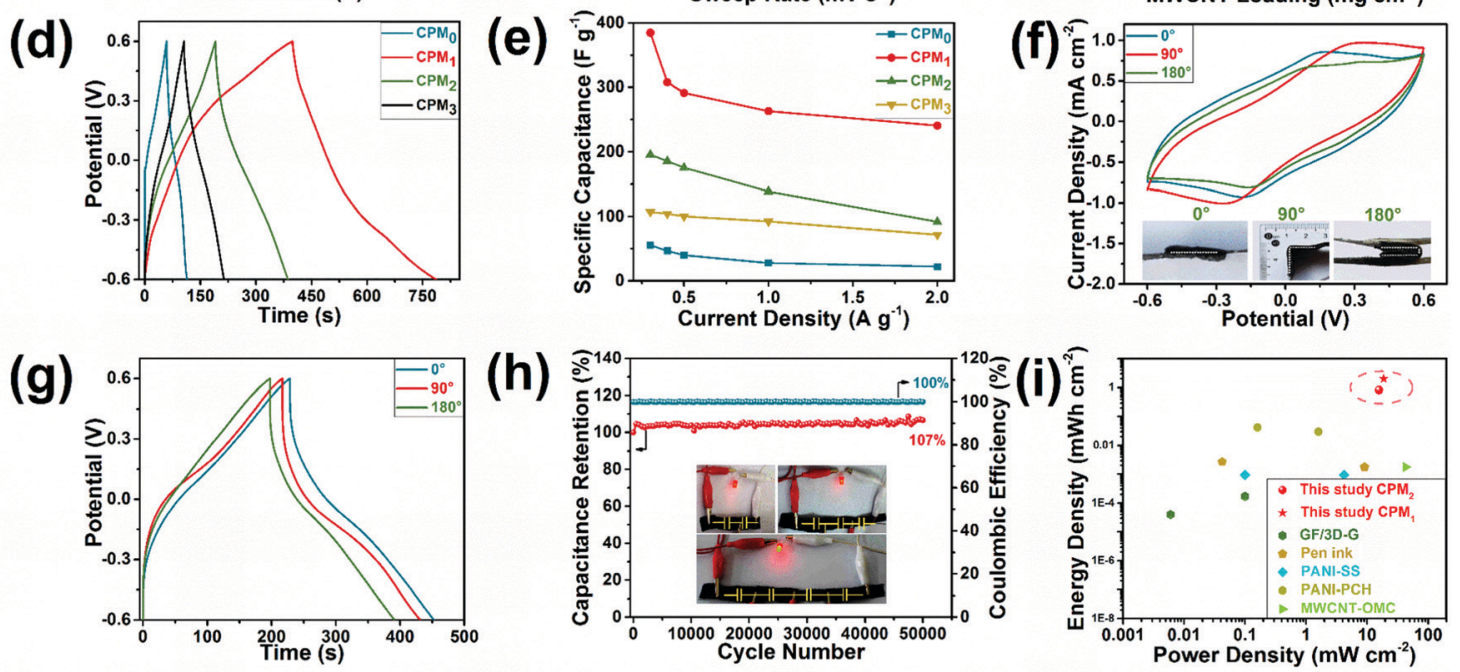

(h)

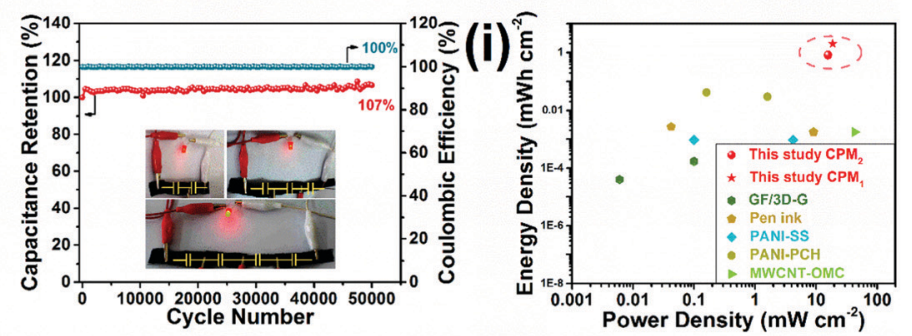

Fig. 4 Electrochemical performance of the integrated FSCs based on single CPM blocks with MWCNT of various weights: (a) CV curves of the CPM ${ }^{-}$ based FSCs at $2 \mathrm{mV} \mathrm{s}^{-1}$, (b) areal specific capacitance at different sweep rates, (c) specific areal capacitance of the FSCs with various mass loadings of MWCNT, (d) GCD curves of the FSCs at $0.3 \mathrm{~A} \mathrm{~g}^{-1}$, (e) gravimetric specific capacitance under different current densities, (f) CV curves for FSCs folded at different angles at $2 \mathrm{mV} \mathrm{s}^{-1}$, (g) GCD curves for FSCs bent in different angles under $0.3 \mathrm{~A} \mathrm{~g}^{-1}$, (h) capacitance retention and Coulombic efficiency of the $\mathrm{CPM}_{2}$-based FSC in the cyclic charge and discharge test under $3 \mathrm{~A} \mathrm{~g}^{-1}$ (inlet: LED lighting with the FSCs), and (i) comparison of Ragone plots of the present and reported supercapacitor devices (GF/3D-G, ${ }^{6}$ Pen ink, ${ }^{33}$ PANI-SS, $^{34}$ PANI-PCH, $^{35}$ and MWCNT-OMC ${ }^{36}$ ).

(Fig. 4c). During the assembly process of the FSCs, MWCNT was coated on the surface after the complete infiltration of the electrolyte. It was adhered by the gel electrolyte and fixed on the composite surfaces through the solid conversion of the electrolyte with the freezing-thawing treatment to ensure full contact between the coated MWCNT and the CPM electrode. Appropriate loading of the MWCNT can be stably stacked in the holes on the CPM surfaces, while a further increase of the MWCNT loading may lead to a loose structure of the electrode materials (Fig. S3d-f, ESI $\dagger$ ) and thus insufficient contact in the electrode material and electrolyte. ${ }^{38}$ Consequently, the MWCNT loading of $7.2 \mathrm{mg} \mathrm{cm}^{-2}\left(\mathrm{CPM}_{3}\right)$ resulted in a lower specific areal capacitance of $0.65 \mathrm{~F} \mathrm{~cm}^{-2}$ than that for the loading of $5.0 \mathrm{mg} \mathrm{cm}{ }^{-2}\left(\mathrm{CPM}_{2}\right)$. Additionally, the $\mathrm{CV}$ curves of the FSCs at various scan rates indicated their ideal capacitive behaviors (Fig. S4, ESI $\dagger$ ). The GCD curves of the FSCs with different MWCNT loadings $\left(\mathrm{CPM}_{0,1,2,3}\right)$ were also recorded under various current densities to evaluate their charge-discharge performance (Fig. S4, ESI $\dagger$ ). The GCD curves for FSCs under the current density of $0.3 \mathrm{~A} \mathrm{~g}^{-1}$ exhibited typical symmetrical triangle-shape charge-discharge profiles, indicating the fast charge/discharge properties of the as-prepared FSCs (Fig. 4d). Similar to the CV results, the GCD curves confirmed that MWCNT loading played a crucial role on the capacitance performance of the FSCs. A maximum gravimetric specific capacitance of $384.69 \mathrm{~F} \mathrm{~g}^{-1}$ was obtained with the sample based on $\mathrm{CPM}_{1}$ composites under a current density of $0.3 \mathrm{~A} \mathrm{~g}^{-1}$. These results also demonstrated that an appropriate MWCNT loading was beneficial to the capacitance performance of the FSCs. However, the optimal MWCNT loading as determined by the GCD results was different from that determined by thee CV results, and thus the appropriate MWCNT loading can be selected according to different specific capacitance requirements (areal, gravimetric, or volumetric). The capacitance performance of FSCs showed a certain degree of stability under various current densities. For example, the gravimetric specific capacitance for $\mathrm{CPM}_{1}$ remained at $240.56 \mathrm{~F} \mathrm{~g}^{-1}$ under a current density of $2 \mathrm{~A} \mathrm{~g}^{-1}$ (Fig. 4e). Notably, GCD curves under various current densities showed no obvious IR drop, and the Coulombic efficiency remained at approximately 100\% during the charge-discharge cycles (Fig. S4, ESI $\dagger$ ). A comparative study confirmed that the electrodes prepared by soaking the electrolyte and subsequently applying MWCNT and PANI showed much lower area and weight specific capacitances than that of the above-mentioned sample (Fig. S5, ESI $\dagger$ ). Therefore, it is necessary to coat the wooden substrate with MWCNT and PANI before electrolyte immersion.

Fig. $4 \mathrm{f}$ and $\mathrm{g}$ illustrates the capacitance performance of the FSCs device under various folding states. The CV curves of the FSCs with different folding angles showed limited changes at a scanning rate of $2 \mathrm{mV} \mathrm{s}^{-1}$. The specific areal capacitance of the FSCs based on $\mathrm{CPM}_{1}$ composites were $0.42,0.39$, and 
$0.34 \mathrm{~F} \mathrm{~cm}^{-2}$ for the folding angles of $0^{\circ}, 90^{\circ}$, and $180^{\circ}$, respectively. The $\mathrm{CV}$ curves show a rectangular shape and notable redox peaks, and the capacitance retention was up to $80.95 \%$ for the folding angle of $180^{\circ}$. The GCD curves of the FSCs in various folding states under a current density of $0.3 \mathrm{~A} \mathrm{~g}^{-1}$ exhibited similar triangular shapes, indicating that the FSCs showed fast charging and discharging performance under the folding states (Fig. 4g). Excellent flexibility and high capacitance retention under folding states provide great possibilities for the application of $\mathrm{CPM}_{X}$-based FSCs in wearable devices.

The consecutive charge-discharge cycles (Fig. 4h) showed the ultrastable cycle performance of the $\mathrm{CPM}_{2}$-based FSCs, and the specific capacitance retention reached $106.68 \%$ after 50000 cycles under a current density of $3 \mathrm{~A} \mathrm{~g}^{-1}$. Moreover, even at an elevated current density $\left(5 \mathrm{~A} \mathrm{~g}^{-1}\right)$, the specific capacitance retention reached 96.41\% (Fig. S4g, ESI $\dagger$ ), although there was a decay of capacitance at the early cycling stage, which may result from the partial pulverization of the thin, top PANI layer and uneven CNT coating. ${ }^{23}$ The slight increase of capacitance at the final stage may result from the effect of a mesoporous structure in the MWCNT and cellulose network on the charge transmission and storage in the FSCs, ${ }^{39}$ as the dominant mesoporous distribution in CPM composites was confirmed by the BET results (Fig. $2 \mathrm{~h}$ ). In addition, the activation of the FSCs via electrochemical cycles could increase the contact area between the electrode material and electrolyte, which could also contribute to the improvement of the specific capacitance. ${ }^{40}$ In addition, the series connection circuit of several FSC devices showed realistic applications, such as lighting up a light-emitting diode (LED) light (Fig. 4h). Although the charging time was slightly extended, the charging voltage of the $\mathrm{CPM}_{2}$-based FSCs after 50000 cycles reached approximately $2.7 \mathrm{~V}$ at a current density of $5 \mathrm{~A} \mathrm{~g}^{-1}$, thereby lighting up a LED successfully (Fig. S4g, ESI $\dagger$ ).
The FSC device based on $\mathrm{CPM}_{1}$ and $\mathrm{CPM}_{2}$ showed a promising energy density of $2.01 / 0.83 \mathrm{~mW} \mathrm{~h} \mathrm{~cm}{ }^{-2}$ at a power density of 18.77/15.67 $\mathrm{mW} \mathrm{cm}^{-2}$ (at a scan rate of $2 \mathrm{mV} \mathrm{s}^{-1}$ ). The comparison on Ragone plots of the present and reported FSC prototypes confirmed the extremely high energy density and power density of the as-prepared FSCs (Fig. 4i). This may be attributed to the abundant porous structure of the cellulose network, which can provide a large place for the deposition of the active substances. The effective deposition of the active substances thus greatly increased the capacitance and energy density per unit area. ${ }^{41}$ Furthermore, the integrated structure was conducive to charge transfer in the supercapacitors and contributed to the improvement of energy storage performance in the present study. ${ }^{23}$

Fig. 5 summarizes the different structures of the conventional supercapacitors and the present integrated all-in-one FSC, the proposed working mechanism of the present FSCs, and the electrochemical performance of the FSCs with various structural prototypes. Generally, two electrodes and a separator between them are needed to assemble a conventional supercapacitor, while only an integrated electrode material was necessary to construct the integrated FSCs in this study (Fig. 5a). The present strategy eliminated the complicated assembly steps of the conventional supercapacitors and reduced the contact resistance between interfaces. Additionally, the working mechanism of the integrated all-in-one FSC was proposed as a hybrid supercapacitor combined electrical double-layer capacitor with faradaic pseudocapacitor according to the literature, as shown in Fig. $5 \mathrm{~b} .{ }^{42}$ During the charging process, the external circuit resulted in the directional movement of electrons and produced a potential difference between the electrodes. In order to maintain the overall electrical neutrality, the anions and cations in the electrolyte moved to

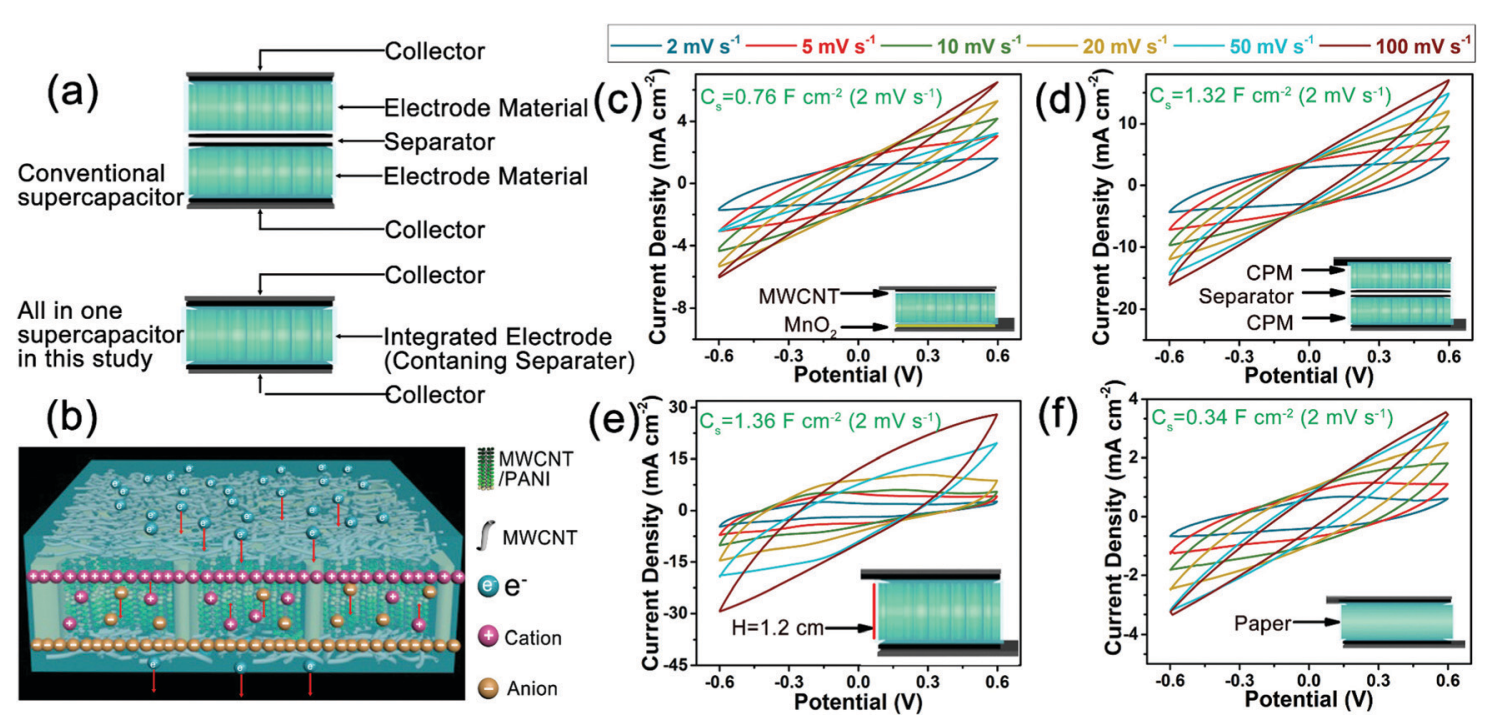

Fig. 5 The structural features, working mechanism, and expansibility of the presented FSCs: (a) different structures of conventional supercapacitors and FSCs in this study, (b) proposed working mechanism of the FSCs, and CV curves under various scan rates for (c) asymmetric FSC, (d) conventional FSC, (e) ultra-thick FSC, and (f) paper based FSC. 
the anode and cathode, respectively, and thus a dense double electric layer was formed in the interface between the MWCNT and electrolyte. ${ }^{40,42}$ Meanwhile, a redox reaction of PANI occurred by losing or gaining electrons, and the resulting directional movement of the anions and cations in the electrolyte produced faradaic pseudocapacitance. ${ }^{43,44}$ The superposition of these two capacitances increased the charge storage capacity of the FSC device. In the discharge process, electrons flowed from the external circuit to the anode, which made the potential difference between the anode and cathode disappear, and the anions and cations on the surface of the electrode material broke away from the attraction of their respective electrodes and migrated back to the electrolyte, thus returning to the previous state of irregular distribution. ${ }^{42-44}$ Additionally, the ion transport capacity of the pure PVA gel electrolyte, PVA gel electrolyte/cellulose network, and PVA gel electrolyte/cellulose paper separator was evaluated by comparing their ionic conductivities with an EIS method in a three-electrode system. The sample of PVA gel electrolyte/cellulose network showed a much higher ionic conductivity $\left(1.85 \times 10^{-2} \mathrm{~S} \mathrm{~cm}^{-1}\right)$ than the pure PVA gel electrolyte $\left(6.33 \times 10^{-3} \mathrm{~S} \mathrm{~cm}^{-1}\right)$ and the PVA gel electrolyte/cellulose paper separator $\left(3.45 \times 10^{-3} \mathrm{~S} \mathrm{~cm}^{-1}\right)$ (Fig. S6, ESI $\dagger$ ). This may be due to the directional porous structure of the cellulose network. The PVA gel electrolyte possessed a relatively compact structure, and the existence of a cellulose network in the PVA gel electrolyte promoted ion transport by providing directional channels for the ion transport. ${ }^{41}$ These results confirmed the above inference on the promotion effect of the cellulose network on the ion transport in the FSCs.

Various structural prototypes of FSCs were also studied to investigate the applicability of the present preparation strategy of FSCs. Manganese dioxide $\left(\mathrm{MnO}_{2}\right)$, as an alternative to MWCNT, and was coated on one side of the CPM composites to assemble asymmetric FSCs. The CV curve at a scan rate of $2 \mathrm{mV} \mathrm{s}^{-1}$ resulted in a higher specific areal capacitance of $0.76 \mathrm{~F} \mathrm{~cm}^{-2}$ than that of the corresponding symmetric FSC based on $\mathrm{CPM}_{2}$ composites (Fig. 5c). The introduction of $\mathrm{MnO}_{2}$ and the asymmetric structure enabled the excellent electrochemical performance of the FSCs, which provides a new idea for designing novel biomass-based supercapacitors with excellent electrochemical performance. ${ }^{45}$ Moreover, we also prepared a conventional-structure FSC device with two $\mathrm{CPM}_{1}$ electrodes and a commercial cellulose separator (Fig. 5d), and, compared with that of the all-in-one $\mathrm{CPM}_{1}$ FSC, a much higher specific areal capacitance of $1.32 \mathrm{~F} \mathrm{~cm}^{-2}$ was achieved through the $\mathrm{CV}$ test at a scan rate of $2 \mathrm{mV} \mathrm{s}^{-1}$; this was mainly attributed to the much higher mass loading (up to $19.18 \mathrm{mg} \mathrm{cm}^{-2}$ ) of active substances (MWCNT and PANI) within unit area due to the utilization of two CPM electrodes. Additionally, the maximum volumetric capacitance of the FSCs based on two pieces of $\mathrm{CPM}_{2}$ was calculated based on the thickness of the whole device (2.2 $\mathrm{mm}$, including the collectors), which was much less than the all-in-one FSC $\left(3.45 \mathrm{~F} \mathrm{~cm}^{-3}\right.$ vs. $\left.5.92 \mathrm{~F} \mathrm{~cm}^{-3}\right)$ based on one piece of $\mathrm{CPM}_{2}(1.2 \mathrm{~mm})$ in this study. The results indicated the integrated structure had the advantages of improving the energy storage performance of FSCs when compared with the conventional structure. An ultra-thick FSC device assembled with a $1.2 \mathrm{~cm}$-thick $\mathrm{CPM}_{1}$ block also showed excellent electrochemical performance (Fig. 5e). The CV curve of the ultra-thick FSC at the scan rate of $2 \mathrm{mV} \mathrm{s}^{-1}$ exhibited an ultrahigh specific areal capacitance of $1.36 \mathrm{~F} \mathrm{~cm}^{-2}$. The ultrahigh mass loading of active substances for the ultra-thick FSC led to the excellent electrochemical performance, and the porous structure of the cellulose network endowed the as-prepared FSCs with an outstanding tunability on the mass loading of active substances. In addition to the wooden cellulose network, the commonly used cellulose paper was used as an electrode supporting material to prepare the all-in-one FSCs (Fig. 5f). The paper-based FSC gave a promising specific areal capacitance of $0.34 \mathrm{~F} \mathrm{~cm}^{-2}$, which was slightly lower than that of the FSC based on $\mathrm{CPM}_{1}$. This may be attributed to the relatively compact structure and the lack of directional channels in the cellulose paper. Based on the above analyses, it can be found that the present strategy for the assembly of FSCs showed great extensibility on the electrode material and supercapacitor structure.

The present results demonstrated that the CPM composite can be used as an important electrode material for highefficiency and ultrastable energy storage applications. Additionally, it should be noted that the hierarchical porous structure of the cellulose network provided an extremely wide space for its performance improvement, which can be used as a platform for the development of green and sustainable high-performance electrode materials.

\section{Conclusion}

This paper presents a new type of integrated FSC with an integrated $\mathrm{CPM}_{X}$ composite block acting as both the electrodes and separator. The combination of the straightforward hierarchical structure of the wood cellulose network with highelectrochemical-active PANI and MWCNT in the $\mathrm{CPM}_{X}$ composites endowed the FSC with a unique structure for ion/electron transmission and an ideal electrochemical energy storage performance; the specific areal capacitance of the as-prepared FSCs based on $\mathrm{CPM}_{2}$ reached up to $0.71 \mathrm{~F} \mathrm{~cm}^{-2}$. The as-prepared FSCs also demonstrated ultrastable cycle performance, and a specific capacitance retention of $106.68 \%$ was achieved after 50000 cycles at a current density of $3 \mathrm{~A} \mathrm{~g}^{-1}$, which showed a significant advantage over reported FSCs. The present study provides a new strategy for the design and construction of high-performance and ultrastable FSCs using renewable natural resources for wearable electronics applications.

\section{Author contributions}

Shaoqiu Ke: conceptualization, methodology, writing - original draft, validation, investigation; Di Xie: conceptualization, methodology, writing - original draft, validation, investigation; Kai Zhang: methodology, investigation; Fangchao Cheng: conceptualization, writing - origin draft, writing - review \& editing, 
supervision, funding acquisition; Yiqiang $\mathrm{Wu}$ : conceptualization, writing - review \& editing, supervision.

\section{Conflicts of interest}

There are no conflicts to declare.

\section{Acknowledgements}

The authors are grateful for the support of the Project Funded by China Postdoctoral Science Foundation (2017M620361), the Natural Science Foundation of Guangxi (2020GXNSFAA297028; 2016GXNSFCA380025), and the Scientific Research Foundation of Guangxi University (XGZ150513).

\section{References}

1 C. Yuan, L. Yang, L. Hou, L. Shen, X. Zhang and X. W. Lou, Energy Environ. Sci., 2012, 5, 7883-7887.

2 D. Wei, S. J. Wakeham, T. W. Ng, M. J. Thwaites, H. Brown and P. Beecher, Electrochem. Commun., 2009, 11, 2285-2287.

3 L. Nyholm, G. Nyström, A. Mihranyan and M. Strømme, Adv. Mater., 2011, 23, 3751-3769.

4 C. Shao, T. Xu, J. Gao, Y. Liang, Y. Zhao and L. Qu, Nanoscale, 2017, 9, 12324-12329.

5 R. Liu, L. Ma, S. Huang, J. Mei, E. Li and G. Yuan, J. Phys. Chem. C, 2016, 120, 28480-28488.

6 J. Ren, W. Bai, G. Guan, Y. Zhang and H. Peng, Adv. Mater., 2013, 25, 5965-5970.

7 J. Wang, L. Dong, C. Xu, D. Ren, X. Ma and F. Kang, ACS Appl. Mater. Interfaces, 2018, 10, 10851-10859.

8 A. Sumboja, C. Y. Foo, X. Wang and P. S. Lee, Adv. Mater., 2013, 25, 2809-2815.

9 X. Lu, Y. Zeng, M. Yu, T. Zhai, C. Liang, S. Xie, M.-S. Balogun and Y. Tong, Adv. Mater., 2014, 26, 3148-3155.

10 Y. Zhang, L. Zhang, K. Cui, S. Ge, X. Cheng, M. Yan, J. Yu and H. Liu, Adv. Mater., 2018, 30, 1801588.

11 M. M. Pérez-Madrigal, M. G. Edo and C. Alemán, Green Chem., 2016, 18, 5930-5956.

12 H.-P. Cong, X.-C. Ren, P. Wang and S.-H. Yu, Energy Environ. Sci., 2013, 6, 1185-1191.

13 Z. Li, Y. Mi, X. Liu, S. Liu, S. Yang and J. Wang, J. Mater. Chem., 2011, 21, 14706-14711.

14 A. R. de Arellano-Lopez, J. Martinez-Fernandez, P. Gonzalez, C. Dominguez, V. Fernandez-Quero and M. Singh, Int. J. Appl. Ceram. Technol., 2004, 1, 56-67.

15 H. Song, S. Xu, Y. Li, J. Dai, A. Gong, M. Zhu, C. Zhu, C. Chen, Y. Chen, Y. Yao, B. Liu, J. Song, G. Pastel and L. Hu, Adv. Energy Mater., 2018, 8, 1701203.

16 F. Jiang, T. Li, Y. Li, Y. Zhang, A. Gong, J. Dai, E. Hitz, W. Luo and L. Hu, Adv. Mater., 2018, 30, 1703453.

17 S. Ke, T. Ouyang, K. Zhang, Y. Nong, Y. Mo, Q. Mo, Y. Wei and F. Cheng, Macromol. Mater. Eng., 2019, 304, 1900112.

18 H. Gao and K. Lian, RSC Adv., 2014, 4, 33091-33113.
19 N. A. Choudhury, S. Sampath and A. K. Shukla, Energy Environ. Sci., 2009, 2, 55-67.

20 M. Kaempgen, C. K. Chan, J. Ma, Y. Cui and G. Gruner, Nano Lett., 2009, 9, 1872-1876.

21 K. Chen, J. Liu, X. Yang and D. Zhang, Mater. Sci. Eng., C, 2017, 78, 520-529.

22 F. Béguin, V. Presser, A. Balducci and E. Frackowiak, Adv. Mater., 2014, 26, 2219-2251.

23 K. Wang, X. Zhang, C. Li, X. Sun, Q. Meng, Y. Ma and Z. Wei, Adv. Mater., 2015, 27, 7451-7457.

24 M. Zhu, J. Song, T. Li, A. Gong, Y. Wang, J. Dai, Y. Yao, W. Luo, D. Henderson and L. Hu, Adv. Mater., 2016, 28, 5181-5187.

25 S. X. Xiong, X. K. Zhang, R. Wang, Y. Z. Lu, H. F. Li, J. Liu, S. Li, Z. Qiu, B. H. Wu, J. Chu, X. Q. Wang, R. L. Zhang, M. Gong and Z. M. Chen, J. Polym. Res., 2019, 26, 90.

26 T.-M. Wu and Y.-W. Lin, Polymer, 2006, 47, 3576-3582.

27 D. Bondeson, A. Mathew and K. Oksman, Cellulose, 2006, 13, 171-180.

28 D. Xu, X. Xiao, J. Cai, J. Zhou and L. Zhang, J. Mater. Chem. A, 2015, 3, 16424-16429.

29 T. Liu, L. Finn, M. Yu, H. Wang, T. Zhai, X. Lu, Y. Tong and Y. Li, Nano Lett., 2014, 14, 2522-2527.

30 D. Ge, L. Yang, L. Fan, C. Zhang, X. Xiao, Y. Gogotsi and S. Yang, Nano Energy, 2015, 11, 568-578.

31 J.-C. Chiang and A. G. MacDiarmid, Synth. Met., 1986, 13, 193-205.

32 H. Huang, J. Yao, L. Li, F. Zhu, Z. Liu, X. Zeng, X. Yu and Z. Huang, J. Mater. Sci., 2016, 51, 8728-8736.

33 Y. Meng, Y. Zhao, C. Hu, H. Cheng, Y. Hu, Z. Zhang, G. Shi and L. Qu, Adv. Mater., 2013, 25, 2326-2331.

34 Y. Fu, X. Cai, H. Wu, Z. Lv, S. Hou, M. Peng, X. Yu and D. Zou, Adv. Mater., 2012, 24, 5713-5718.

35 Y. Fu, H. Wu, S. Ye, X. Cai, X. Yu, S. Hou, H. Kafafy and D. Zou, Energy Environ. Sci., 2013, 6, 805-812.

36 W. He, J. P. Li, J. X. Tian, H. Jing and Y. J. Li, Polym. Compos., 2018, 39, 537-543.

37 W. Zhao, Y. Li, S. Wu, D. Wang, X. Zhao, F. Xu, M. Zou, H. Zhang, X. He and A. Cao, ACS Appl. Mater. Interfaces, 2016, 8, 34027-34033.

38 W. Liu, S. Wang, Q. Wu, L. Huan, X. Zhang, C. Yao and M. Chen, Chem. Eng. Sci., 2016, 156, 178-185.

39 L. Hao, X. Li and L. Zhi, Adv. Mater., 2013, 25, 3899-3904. 40 D. Shin, Y. Ko and J. Cho, RSC Adv., 2016, 6, 21844-21853. 41 Y. Wu and C. B. Cao, Sci. China: Mater., 2018, 61, 1517-1526. 42 S. Goswami, G. R. Dillip, S. Nandy, A. N. Banerjee, A. Pimentel, S. W. Joo, R. Martins and E. Fortunato, Electrochim. Acta, 2019, 316, 202-218.

43 W. Chen, R. B. Rakhi and H. N. Alshareef, J. Phys. Chem. C, 2013, 117, 15009-15019.

44 L. B. Chen, Y. R. Chen, J. F. Wu, J. W. Wang, H. Bai and L. Li, J. Mater. Chem. A, 2014, 2, 10526-10531.

45 N. Choudhary, C. Li, J. Moore, N. Nagaiah, L. Zhai, Y. Jung and J. Thomas, Adv. Mater., 2017, 29, 1605336. 